\title{
Neue Veröffentlichungen der beteiligten Institute
}

Institut für Asienkunde, 2 Hamburg 13, Rothenbaumchaussee 32

„Schriften des Instituts für Asienkunde“:

Band 22 Paul W. Wilm:

Die Fruchtbarkeit und Ertragsleistung

Nordchinas bis 1949

Wiesbaden 1968, 258 S., 48,- DM

Es handelt sich um eine agrarwissenschaftliche Untersuchung aufgrund mehrähriger Feldstudien, die eine Fülle von Material zur Beurteilung heutiger agrotechnischer Maßnahmen in der Volksrepublik China bietet.

„Mitteilungen des Instituts

für Asienkunde":

Heft 23 Karl Jettmar:

Zur wissenschaftspolitischen Funktion von Außen- und Kontaktinstituten in Entwicklungsländern 1967, 19 S.

Eine Betrachtung zur Position wissenschaftlicher Institute und der Zusammenarbeit $\mathrm{zwischen}$ solchen Instituten im Rahmen der Entwicklungshilfe.

Band 23 Brunhild Staiger:

Das Konfuzius-Bild

im kommunistischen China

Wiesbaden 1969, 143 S., 20,- DM

Ausgehend von der Reformbewegung und der 4. Mai-Bewegung gibt diese Arbeit eine Analyse des Konfuzius-Bildes der Volksrepublik China.

Band 24 Sung-Jo Park:

Die Wirtschaftsbeziehungen zwischen

Japan und Korea 1910-1968

Wiesbaden 1969, 268 S., 111 Tab., 3 K. 50,- DM.

Darstellung der Entwicklung der ökonomischen Beziehungen von der kolonialen Epoche bis zum japanisch-koreanischen Normalisierungsvertrag.

Sonderveröffentlichung:

Wolfgang Bartke:

Das Politbüro des 8. Zentralkomitees der Kommunistischen Partei Chinas

Wiesbaden 1969, 165 S., 46 Tab., 10 Faltkarten, 40,- DM.

Darstellung des Herkommens, der persönlichen Hintergründe und der gegenseitigen Beziehungen der Mitglieder des letzten Politbüros vor der Kulturrevolution.

\section{Heft 24 Klaus Röh: \\ Zum Arbeitsverhalten der indischen Belegschaft in Rourkela 1968, 94 S.}

Beurteilung des Verhaltens indischer Arbeiter aufgrund von Interviews und Feldstudien.

Heft 25 Oskar Weggel:

Die chinesischen Revolutionskomitees oder

der Versuch, die Große Kultur-

revolution durch Parzellierung zu retten 1968, 122 S.

Ein Beitrag zur Deutung der Vorgänge im Rahmen der Großen Proletarischen Kulturrevolution.

Heft 26 Volker Matthies:

China und Afrika

1969, 78 S.

Úberblick über die Entwicklung der diplomatischen Beziehungen, wichtige Personenkreise und Institutionen im Rahmen der chinesischen Afrika-Politik. 
Heft 27 Dieter Heinzig:

Die Krise der Kommunistischen Partei Chinas in der Kulturrevolution

1969, 48 S.

Ein Beitrag zur Deutung der Vorgänge im Rahmen der Großen Proletarischen Kulturrevolution.

Heft 28 Klaus Wenk:

Die neue Verfassung Thailands 1969, 36 S.

(gleichzeitig erschienen in Heft 1 (1969) der Zeitschrift „Verfassung und Recht in Übersee")

Deutsches Institut für Afrika-Forschung e. V., 2 Hamburg 36, Schleusenbrücke 1

Hamburger Beiträge zur Afrikakunde

Band 4 Wolfgang Heidelberg

Grundzüge des Niederlassungsrechts in den afrikanischen Staaten. Teil 2

(englischsprachige Gebiete)

Hamburg 1969, 216 S., Kart. 22,- DM.

In dieser Schrift werden die für den privaten Investor in 11 Staaten des britischen Rechtskreises in Afrika relevanten Rechtsvorschriften erstmalig im $\mathrm{Zu}$ sammenhang dargestellt. Den Schwerpunkt der Untersuchung bilden die Frage des Schutzes vor Enteignung und die Garantien und Vergünstigungen für Investoren.

Band 9 Hansgünter Schönwälder Die Kakaowirtschaft Westafrikas Hamburg 1969, 232 S., kart., 24,- DM.

In dieser Arbeit werden die ökologischen und ökonomischen Aspekte der Kakaowirtschaft in ihrem wichtigsten Produktionsgebiet behandelt.

Band 10 Christoph Becker

Kano, eine afrikanische Großstadt (im Druck).

Am Beispiel der traditionsreichen nordnigerianischen Stadt wird der Wandel zu einem modernen afrikanischen $\mathrm{Ge}-$ meinwesen dargestellt.
Heft 29 C. L. Yu:

Die Möglichkeiten und Grenzen des chinesischen Außenhandels 1969, 49 S.

Die „Schriften . . . und die Sonderveröffentlichung sind im Verlag Otto Harrassowitz erschienen und über den Buchhandel zu beziehen. Die „Mitteilungen" sind im Eigenverlag des Instituts erschienen.

\section{Afrika Spectrum}

Heft 11969

Recht und Rechtspraxis in Afrika 80 S., $8,50 \mathrm{DM}$

Dieses Heft ist Verfassungsproblemen und insbesondere der Verfassungswirklichkeit in einigen ausgewählten afrikanischen Staaten gewidmet. Außerdem enthält es den nach Stichwörtern gegliederten Jahresindex 1968 der Dokumentation aus afrikanischen Gesetzesblättern.

\section{Heft 21969}

Afrikaforschung in der Bundesrepublik 130 S., 8,50 DM

Hier wird ein Zwischenbericht über die Forschungsprogramme und die Dokumentationsarbeit westdeutscher AfrikaInstitute gegeben.

Afrika Branchenberichte (1)

Per Klümper

Die Baumwoll-, Textil- und Schuhproduktion in Afrika

Hamburg 1969, 128 S., kart., 10,- DM. Mit dieser Untersuchung wird der erste Band einer Reihe vorgelegt, in der die relevanten Daten über die für den Investor und den Außenhandel wichtigen Industriebranchen im tropischen Afrika zusammengestellt sind. 
Institut für Iberoamerika-Kunde, 2 Hamburg 36, Alsterglacis 8

Schriftenreihe des Instituts (Ưbersee-Verlag, Hamburg)

Band 9 Albrecht Kruse-Redenacker

Die interamerikanische

Entwicklungsbank

Externe Entwicklungsfinanzierung im internationalen Kredit- und Kapitalverkehr

1968, 116 S., 13,80 DM.

Entstehungsgeschichte, Aufbau und Wirkungsweise der IAEB (BID) vor dem Hintergrund der allgemeinen Situation und der spezifischen Probleme der lateinamerikanischen Länder bei der externen Entwicklungsfinanzierung.

Besondere Berücksichtigung finden die wirtschaftlichen Beziehungen zwischen Lateinamerika und den USA.

Vollständige Übersetzung der Gründungsabkommen sowie zahlreiche Tabellen.

Band 10 Hans-Rudolf Horn

México: Revolution und Verfassung

Der mexikanische Weg zur politischen Stabilität

1969, 164 S., 15,60 DM

México hat es als bisher einziges Land Iberoamerikas verstanden, die in den iberoamerikanischen Ländern bestehende Lücke zwischen formalem Verfassungsrecht und politischer Wirklichkeit auszufüllen. Die 1917 beschlossene Verfassung ist zum Grundgesetz geworden für die bis heute erfolgreiche evolutionäre Entwicklung Méxicos.

Die Verfassung Méxicos wird aus ihrer Entstehungsgeschichte heraus behandelt, wie auch das Verhältnis der Staatsgewalt zum Militarismus, zum Grundrechtschutz, zur Kirche und zu Agrarreform und entwicklungspolitischen Aufgaben.

Band 11 Alexander Bohrisch

Probleme privater

Auslandsinvestitionen in México 1969, 132 S., 15,60 DM

Neben Argentinien und Brasilien ist México neuerdings das Land, das wegen seines stetigen Wirtschaftswachs- tums umfangreiche Auslandsinvestitionen auf sich zieht. Der Autor schildert, ausgehend von der mexikanischen Industrialisierungspolitik, die Haltung, dieses Landes gegenüber dem ausländischen Privatkapital und gibt einen Úberblick über wichtige rechtliche $\mathrm{Be}$ stimmungen. In einem besonderen $\mathrm{Ka}$ pitel werden die aus eingehenden persönlichen Befragungen ermittelten Erfahrungen ausländischer Unternehmer in México analysiert.

\section{Band 12 Robert. K. Furtak}

Revolutionspartei und politische

Stabilität in México

1969, 136 S., 15,60 DM

Zum beherrschenden Faktor auf dem Wege zur politischen und wirtschaftlichen Stabilität wurde ein Parteiwesen, das auf eigene "mexikanische“ Weise gestaltet wurde, nämlich zunächst durch eine von oben gegründete und mit dem Staatsapparat intensiv verknüpfte führende Partei, die sich schon in ihrem Namen die Fortführung der in der Verfassung von 1917 verankerten Ziele setzte: Partido Revolucionario Institucional.

Diese Partei verkörpert eine Mischung von autoritären und demokratischen Elementen und Prinzipien und ist nur als eine typische Erscheinung der "mexicanidad“ zu begreifen. Die Arbeit vermittelt eine ausführliche Analyse dieser Partei mit Blick auf die übrigen bestehenden Parteien und gibt zugleich wertvolle Einblicke in das erstaunliche Wachsen der politischen Stabilität dieses Landes und die Überwindung der sonst in Iberoamerika so typischen, verhängnisvollen Zersplitterung.

Band 13 Wolfgang König

Devisenkurspolitik in Lateinamerika 1969, 148 S., 15,60 DM

Stabilisierung der Währung und Ausgleich der Zahlungsbilanz sind zentrale Probleme der Wirtschafts- und Entwicklungspolitik in Lateinamerika. Da- 
bei spielt das Verhältnis der nationalen Devisenkurspolitik zur weltweiten währungspolitischen Zusammenarbeit und zum Internationalen Währungsfonds eine wichtige Rolle, deren Faktoren und Auswirkungen bisher selbst in Fachkreisen kaum bekannt sind. Der Autor, der die Hintergründe und verschiedenartigen Aspekte dieser Problematik in mehrjährigen Studien in Lateinamerika und den USA eingehend untersucht hat, stellt sowohl die theoretischen Zusammenhänge als auch die praktische Seite dieser komplexen Problematik dar, wobei insbesondere auch die Frage der multiplen Wechselkurse behandelt wird.

\section{Band 14 Jürgen Westphal (Hrsg.)}

Neue Wege der Berufsausbildung

in Lateinamerika

1969, 120 S., 13,80 DM

Der Bereich der Bildung und Ausbildung hat in allen lateinamerikanischen Ländern, aber auch im Rahmen der deutschen Entwicklungspolitik für Lateinamerika in den letzten Jahren erheblich an Bedeutung gewonnen. Man ist sich heute in Kreisen der Entwicklungspolitik und Wissenschaft darüber klar, daß wirtschaftliche und soziale Weiterentwicklung in Lateinamerika neue wirksame Initiativen im Bildungswesen zur Voraussetzung hat. Die vorliegende Broschüre befaßt sich mit einem wichtigen Teilbereich des Bildungswesens, und zwar hauptsächlich mit der gewerblichen Berufsausbildung in sechs lateinamerikanischen Ländern. Alle Autoren der verschiedenen Beiträge dieser Sammlung hatten Gelegenheit, gründliche Untersuchungen in Lateinamerika vorzunehmen. Hier wird folglich eine Informationsquelle vorgelegt, die aus erster Hand über die Gegenwartsproblematik der Berufsausbildung in Lateinamerika unterrichtet.

Band 15 Miguel S. Wionczek

Lateinamerika und das ausländische

Kapital

1969, 100 S., 13,80 DM.

Die immer brennender werdende Frage nach der Rolle der privaten ausländi- schen Investitionen im Entwicklungsprozeß Lateinamerikas wird von einem international anerkannten Wirtschaftswissenschaftler behandelt, der beide Seiten des Problems - die Zielsetzung und Erwartungen der Lateinamerikaner wie die Interessen der Unternehmer in den Industrieländern - aus jahrzehntelanger Erfahrung kennt und zu beurteilen versteht. Jenseits einer einseitigen oder gar demagogischen Betrachtungsweise werden die vielschichtigen Probleme einem nüchternen Studium unterzogen und dabei die bereits erkennbaren Tendenzen der künftigen Haltung Lateinamerikas gegenüber dem Auslandskapital aufgezeigt. Die Broschüre dürfte sowohl für Wirtschaftswissenschaftler und Politologen, die sich mit Entwicklungsländern und ihrem Verhältnis zu den Industrienationen befassen, als auch für Unternehmer, die Auslandsinvestitionen planen, von größtem Interesse sein.

Außerhalb der Schriftenreihe sind erschienen:

Karl M. Helbig

Die Wirtschaft Zentralamerikas

Kartographisch dargestellt und erläutert von Karl M. Helbig 132 S., $19,80 \mathrm{DM}$

Die Wirtschaftskarte ist teils nach dem vorhandenen Kartenmaterial, mehr jedoch nach Beobachtungen des Verfassers während ausgedehnter geographischer Reisen in Gesamt-Zentralamerika angefertigt. Einschlägige Wirtschaftsberichte zentral-amerikanischer und deutscher Institutionen sind hinzugezogen worden.

Im Anhang: Angaben über Quellenmaterial, länderkundliche Hinweise und Tabellen. In Tasche gesondert: Mehrfarbig angelegte Wirtschaftskarte $1: 2500000$ im Format $70 \times 50 \mathrm{~cm}$ sowie Karte der Bevölkerungsdichte (1960) 1:1000 000 im Format 40x25 cm. Außerdem 16 im Texteil eingebaute Kunstdruckseiten mit 62 Abbildungen nach Aufnahmen des Verfassers. 
Friedrich Wehner (Hrsg.)

Idee und Wirklichkeit in Iberoamerika

Beiträge zur Politik und

Geistesgeschichte

1969, 131 S., 25,- DM

(Verlag Hoffmann und Campe, Hambg.)

In Iberoamerika treffen Traditionen zusammen mit dem Bestreben und Verlangen auch teilzuhaben an den Früchten der modernen Zivilisation in der industriellen Gesellschaft.

Die Länder Iberoamerikas haben sich vor 15 Jahren zwar politisch befreit, die Menschen in diesen Ländern sind jedoch stark in der iberischen Erbschaft verwurzelt. Aus dieser Situation ergeben sich Widersprüche, die den Iberoamerikanern das Leben nicht leichter machen und den Außenstehenden das Verständnis erschweren. Dier hier vorliegende Zusammenfassung von Arbeiten aus verschiedenen Disziplinen über die geistesgeschichtlichen Voraussetzungen dürfte das Verständnis für die Eigenart Iberoamerikas mehr als einzelne Fachforschungen fördern.

Inhalt: Vorwort des Herausgebers; Rudolf Grossmann, Das Erbe der Mönche und Conquistadoren; Friedrich Wehner, Der Konflikt zwischen spanischer und liberaler Staatsauffassung in HispanoAmerika; Inge Wolff, Desintegration und Staatenbildung in Hispanoamerika, 1810 bis 1840; Günter Kahle, Historische Bedingtheit der Diktatur in Lateinamerika; Adolf Meyer-Abich, Der Caudillo in seiner historischen Gestalt; Günter Kahle/Friedrich Wehner, Das Militär in der Politik in Lateinamerika; Carlos Stoetzer, Die geistigen Grundlagen der spanisch-amerikanischen Unabhängigkeit; Peter Schenkel, Die ideologische Herausforderung Lateinamerikas durch die cubanische Revolution.

Hamburger Gesellschaft für Völkerrecht und Auswärtige Politik

2 Hamburg 13, Mittelweg 186

Klaus Peters

Die Herrschaftsordnung von Buganda

Ein Weg zu institutionalisierter Herrschaft im alten Afrika

(Schriftenreihe zur Auswärtigen Politik, Bd. 5)

Kommissionsverlag: Hamburger Gesellschaft für Völkerrecht und Auswärtige Politik, Hamburg

1968 VII, 180 S., 17,50 DM

Das Buch stellt am Modell eines afrikanischen Großreiches der alten Zeit die von Europa unbeeinflußte Form eines vorstaatlichen afrikanischen Gemeinwesens dar. Darüber hinaus vergleicht es die afrikanischen Herrschaftstechniken und gesellschaftlichen Entwicklungen mit europäischen und asiatischen Wegen zu staatlicher Ordnung und zeigt die Gemeinsamkeiten und Unterschiede dieser Entwicklungen auf. Es betrachtet schließlich kritisch die Bewährung der afrikanischen Institutionen in der Auseinandersetzung mit europäischen Kräften am Ende des 19. Jahrhunderts und umreißt die Einfügung der überlieferten Institutionen in den neuen Staat Uganda.

Der Verfasser, studierter Jurist und passionierter Ethnograph, räumt durch dieses Buch gründlich mit der Vorstellung auf, daß Afrika südlich der Sahara eine Region ohne Geschichte sei.

Forschungsstelle für Völkerrecht und ausländisches öffentliches Recht der Universität Hamburg, 2 Hamburg 13, Mittelweg 186

Dietrich Steinicke

Quellenindex zur Cubakrise

Eine Zusammenstellung aller im Zusammenhang mit der Quarantäne Cubas im Jahre 1962 bekanntgewordenen Doku- mente mit Inhaltsangaben sowie mit einem völkerrechtlichen Schlagwortverzeichnis. Zusammengestellt von Dietrich Steinicke unter Mitwirkung von Wolfdieter Harbeck. 
(Werkhefte der Forschungsstelle, Bd. 12)

Kommissionsverlag: Alfred Metzner Verlag, Frankfurt am Main und Berlin 1969, VIII, 400 S., 38,20 DM

Die Bearbeiter haben versucht, das gesamte Dokumentenmaterial, das im $\mathrm{Zu}$ sammenhang mit der Quarantäne Cubas im Oktober/November 1962 entstanden ist, zu erfassen, um eine objektive und exakte Analyse der dabei aufgetauchten völkerrechtlichen Fragen zu ermöglichen. Berücksichtigt werden die Dokumente über die Vorbereitung der Quarantäne, über deren vertragliche Grundlagen und über die Reaktionen der betroffenen Staaten. Eine chronologische und eine alphabetische Übersicht nach Ländern erleichtert das Auffinden eines einzelnen Dokuments. Damit sich der Bearbeiter von völkerrechtlichen Fragen im Zusammenhang mit der $\mathrm{Cu}-$ bakrise aber auch ein Bild von der Bedeutung des einzelnen Dokuments machen kann, sind den bibliographischen Angaben Stichworte vorangestellt, die den Inhalt unter völkerrechtlichen $\mathrm{Ge}-$ sichtspunkten vollständig zu erfassen suchen. Den Abschluß bildet eine Zusammenstellung des bisher erschienenen völkerrechtlichen Spezialschrifttums.

\section{Dieter Schröder \\ Die Konferenzen der „Dritten Welt“ \\ Solidarität und Kommunikation \\ zwischen nachkolonialen Staaten \\ (Darstellungen zur Auswärtigen Politik Bd. 7) \\ Kommissionsverlag: Hamburger \\ Gesellschaft für Völkerrecht \\ und Auswärtige Politik, Hamburg \\ 1968, X, 343 S., 42,- DM}

Die Darstellung zeichnet anhand von mehr als hundert großen und einer Vielzahl kleiner Konferenzen ein Bild der „Dritten Welt". Aus den häufig unveröffentlichten und manchmal nur durch Presseberichte rekonstruierten Dokumenten von Konferenzen und Kongressen, die in den letzten hundert Jahren Politiker Asiens, Afrikas und Lateinamerikas zusammengeführt haben, tre- ten die Hauptlinien der Solidarität und die Probleme einer Kommunikation zwischen Politikern und Staaten der vielgestaltigen vormals kolonialen Kontinente hervor. Es wird gezeigt, daß Programme, die heute im Mittelpunkt der Diskussion stehen, etwa die Forderung nach Entwicklungshilfe, schon im 19. Jahrhundert in Asien formuliert worden sind; es wird aber auch deutlich gemacht, vor welchen Schwierigkeiten die nachkolonialen Staaten heute bei der Formulierung einer gemeinsamen Plattform für ihre Politik gegenüber europäischen Staaten stehen.

\section{Knud Krakau}

\section{Die kubanische Revolution und}

die Monroe-Doktrin

(Untersuchungen zur Auswärtigen Politik, Bd. 5),

Alfred Metzner Verlag, Frankfurt a. M. und Berlin

1968, 220 S., 44,- DM

Die Vereinigten Staaten sind durch die kubanische Revolution von 1958/59 innerhalb ihres engsten Einflußbereiches einem politischen System konfrontiert worden, das von der Mehrheit ihrer Bürger als „unamerikanisch" abgelehnt wird. Die Reaktionen der USA auf diese Herausforderung werden in dem vorliegenden Buch zum ersten Male unter dem Gesichtspunkt der Monroe-Doktrin behandelt, die - beinahe 150 Jahre nach ihrer Verkündung - als fertiges Denkmodell immer noch einen bestimmenden Faktor der US-Außenpolitik darstellt.

Ihrem Wesen nach ist die Monroe-Doktrin stets eine Schutzdoktrin gewesen. Sie will sowohl die militärische und politische wie auch die ideologische Machtausübung fremder politischer Systeme in Amerika verhindern. So wurde sie auch mitbestimmend bei der Isolierung Kubas und bei dem Versuch, die Ausbreitung der kubanischen Revolutionsideologie in ganz Amerika einzudämmen und das durch Kuba ermöglichte militärische Eindringen außeramerikanischer Mächte nach Amerika abzuwehren. 